\title{
Effects of omalizumab therapy on peripheral nerve functions: short observational study
}

\author{
Goknur Ozaydın Yavuz¹, Abdullah Yılgör², Ibrahim Halil Yavuz¹, Aysel Milanlığlu², Vedat Çilingir², Aydın Çağaç², \\ Murat Ozturk ${ }^{1}$, Serap Gunes Bilgili ${ }^{1}$
}

1Department of Dermatology, Faculty of Medicine, Van Yuzuncu Yil University, Van, Turkey

2Department of Neurology, Faculty of Medicine, Van Yuzuncu Yil University, Van, Turkey

Adv Dermatol Allergol 2019; XXXVI (2): 211-216

DOI: https://doi.org/10.5114/ada.2018.74834

\begin{abstract}
Introduction: Peripheral neuropathy (PN) is a common neurological condition causing symmetrical and diffuse damage in nerves. The etiology of PN includes systemic diseases, toxic exposure, medications, infections, and hereditary diseases. Omalizumab is a humanized monoclonal anti-lgE antibody that exerts its activity by binding to free IgE in circulation.

Aim: To investigate the relationship between omalizumab and peripheral neuropathy.

Material and methods: The study included 30 patients who underwent omalizumab therapy (Xolair) due to the diagnosis of chronic urticaria. A detailed neurological and physical examination was performed in each patient both before and 3 months after the therapy. Electrophysiological examination was also performed using a Medelec Synergy instrument.

Results: The 30 patients included 8 (26.7\%) men and 22 (73.3\%) women with a mean age of $37.5 \pm 14.14$ years. No serious side effect of the medication was detected in any patient although local wound irritation occurred in $3(10 \%)$ patients. Moreover, no change occurred in the pre-treatment Neuropathy Symptom Score (NSS) or Neurological Disability Score (NDS) of the patients and no pathological values that could result in neuropathy were observed during motor/sensory nerve conduction. However, significant changes were detected in the sensory and motor components of the nerves with regards to pre- and post-treatment values.

Conclusions: Omalizumab therapy caused no peripheral neuropathy in any of our patients but altered the latency, amplitude, and velocity values of the peripheral nerves.
\end{abstract}

Key words: chronic urticaria, omalizumab, neuropathy.

\section{Introduction}

Peripheral neuropathy (PN) is one of the most common neurological conditions, causing symmetrical and diffuse damage in nerves. The etiology of PN includes systemic diseases, toxic exposure, medications, infections, and hereditary diseases. The most common medications associated with PN include amiodarone, chloroquine, hydralazine, lithium, metronidazole, phenytoin, isoniazid, statins, and vincristine. In addition, biological agents including infliximab and adalimumab have been reported in recent case studies. The prevalence of PN has been reported to be as high as $2.4 \%$ in the general population and to be $26.4 \%$ in patients with diabetes mellitus [1-4].
Immunoglobulin E (IgE) plays a central role in the pathogenesis of allergic conditions. Therefore, anti-lgE therapies play a key role in the treatment of allergic diseases such as asthma [1-5]. Omalizumab is a humanized monoclonal anti-lgE antibody. Omalizumab exerts its activity by binding to free IgE in circulation, thereby inhibiting the binding of IgE to its high-affinity receptors (FcERI) found on mast cells and basophils, ultimately reducing the expression of mediators in mast cells. Omalizumab is also an important treatment option particularly for severe asthma and resistant chronic urticaria [6-8].

On the other hand, although histamine is the most important mediator expressed in mast cells, neuropeptides such as nerve growth factor (NGF) are also expressed in these cells [9]. A previous study reported that

Address for correspondence: Goknur Ozaydın Yavuz MD, Department of Dermatology, Faculty of Medicine, Van Yuzuncu Yil University, 65100 Van, Turkey, phone: +90 505 4753362, +90 5054753362, fax: +90 5054753362, e-mail: goknuroz1@gmail.com Received: 27.02.2018, accepted: 12.03.2018. 
mast cell activation led to an increase in the production and secretion of neuropeptides and the excitability of sensory nerves [10]. A recent study evaluated the effectiveness of omalizumab at 52 weeks and showed that omalizumab is a safe drug although it had several side effects including headache, injection site reaction, myalgia, lethargy, nausea, dizziness, weight gain, and arthralgia [11]. However, another study showed that omalizumab therapy resulted in optic neuritis when used for the treatment of bronchial asthma in 2 patients with Churg-Strauss syndrome [12]. Similarly, Lieberman et al. compared omalizumab therapy with placebo therapy and reported that local skin reactions occurred in $44 \%$ of the patients treated with omalizumab [13].

In our patients, we also performed omalizumab therapy for the treatment of chronic urticaria. However, the complaints of pain and weakness in the extremities gradually increased in our patients and thus we could not be sure whether these complaints resulted from local irritation or a neurological condition caused by omalizumab, mainly because the drug was administered in two separate infusions with 150 mg flacons. Moreover, these conditions may be a result of peripheral nerve injury caused by the inhibition of mast cells that leads a reduction in the expression of neuromediators.

\section{Aim}

In this study, we aimed to evaluate the relationship between omalizumab and peripheral neuropathy.

\section{Material and methods}

The study included 30 patients who underwent omalizumab therapy (Xolair) due to the diagnosis of chronic urticaria. Age, gender, socioeconomic status, and family history were recorded for each patient. Omalizumab was subcutaneously administered at $300 \mathrm{mg} /$ day for 28 days (total 4 times: 1, 29, 57, 85 days) in the Dermatology Department. To determine the presence of other factors that may affect peripheral nerve function, additional tests were performed, including complete blood count, sedimentation rate, liver and kidney function tests, urine analysis, thyroid hormones, vitamin $\mathrm{B}_{12}$ level, folic acid level, and serologic tests. In addition, neuroradiological imaging was performed as needed. Exclusion criteria included neurological symptoms and signs, diabetes mellitus, connective tissue disease, hepatic, renal, and thyroid diseases, amyloidosis, heart failure, alcohol abuse, corticosteroid use, cervical disc hernia, and malignancy. A detailed neurological and physical examination was performed in each patient before and three months after the therapy. Neurological symptoms were scored using the Neuropathy Symptom Score (NSS) and Neurological Disability Score (NDS). Following the neurological examination, electrophysiological exami- nation was performed using a Medelec Synergy instrument (Oxford Instruments, Surrey, UK) with standard neurographic procedures, and the results were evaluated according to the American Diabetes Association (ADA) Diabetic Neuropathy Guidelines in the Neurology Department [14]. The measurements were performed $24 \mathrm{~h}$ before and 90 days after omalizumab therapy. Room temperature was kept at $22-24^{\circ} \mathrm{C}$ and the temperature of the extremity was kept at $34^{\circ} \mathrm{C}$ and it was heated as needed. Nerve conduction tests were performed in two motor and two sensory nerves (median and ulnar nerves) in the upper extremities and in two motor (tibial and common peroneal nerve) and two sensory nerves (sural and superficial perneal sensory nerves) in the lower extremities. Pre- and post-treatment latency $(\mathrm{ms})$, amplitude $(\mathrm{mV})$, and velocity $(\mathrm{m} / \mathrm{s})$ values were compared for each nerve. Nerve conduction velocity was measured using the orthodromic method and nerve conduction was performed at supramaximal intensity to achieve the highest amplitude. Presence of an axonal pathology and demyelination in the nerves was defined as decreased sensory/motor nerve action potential amplitude and slowing of sensory/motor nerve conduction velocity. Polyneuropathy was defined as the presence of two or more abnormalities detected in electrophysiological examination. The study was approved by the local ethics committee and informed consent was obtained from each patient (Number: Y.Y.U: 2017/ 02).

\section{Statistical analysis}

Data were analyzed using IBM SPSS for Windows, Version 20.0 (Armonk, NY: IBM Corp.). Normal distributions of variables were determined by histogram and/or by the Kolmogorov-Smirnov/Shapiro-Wilk test. Descriptive statistics were expressed as mean, standard deviation (SD), median, minimum, and maximum. Numerical variables were compared using the paired sample $t$-test for data with normal distribution and the Wilcoxon signed-rank test for data with non-normal distribution. Spearman's correlation coefficient was used to assess the correlation between variables. A $p$-value of $<0.05$ was considered significant.

\section{Results}

The 30 patients included 8 (26.7\%) men and 22 (73.3\%) women with a mean age of $37.5 \pm 14.14$ years (Table 1). No serious side effect of the medication was observed in any patient although local wound irritation occurred in $3(10 \%)$ patients. No change occurred in the pre-treatment NSS and NDS scores of the patients. Moreover, no pathological values that could result in neuropathy were observed during motor/sensory nerve conduction. Nevertheless, significant changes were detected in the sensory and motor components of the nerves with regards to pre- and post-treatment values. 
A comparison of pre- and post-treatment latency, amplitude, and velocity values of the sensory component of the median nerves indicated no significant difference between the pre- and post-treatment latency and velocity values ( $p>0.05)$. However, post-treatment amplitude values (mean: $23.03 \pm 4.71$ ) were significantly lower than pre-treatment values (mean: $24.61 \pm 5.55)(p=0.024)$ (Table 2).

No significant difference was found between the pre- and post-treatment latency and velocity values of the sensory component of the ulnar nerves $(p>0.05)$. Nevertheless, post-treatment amplitude values (mean: $18.71 \pm 5.34)$ were significantly lower than pre-treatment values (mean: $20.11 \pm 5.61)(p=0.030)$ (Table 3 ).

In the sensory component of the sural nerves, no significant difference was found between the pre- and post-treatment amplitude values $(p>0.05)$. However, post-treatment latency values (mean: $2.12 \pm 0.24$ ) were significantly higher than pre-treatment values (mean: $1.96 \pm 0.21)(p=0.009)$, whereas post-treatment velocity values (mean: $46.34 \pm 3.60$ ) were significantly lower than pre-treatment values (mean: $48.14 \pm 7.09)(p=0.001)$ (Table 4).

In the sensory component of the superficial peroneal nerves, no significant difference was found between preand post-treatment latency values $(p>0.05)$, whereas post-treatment amplitude and velocity values (mean: $14.00 \pm 3.02$ and $47.20 \pm 3.36$, respectively) were significantly lower than pre-treatment values (mean: 16.45 \pm 4.05 and $49.34 \pm 3.36$, respectively) ( $p<0.001$ for both) (Table 5).

On the other hand, a comparison of pre- and posttreatment latency, amplitude, and velocity values of the motor component of the median nerves indicated no significant difference between the pre- and post-treatment latency values $(p>0.05)$ although post-treatment amplitude and velocity values (mean: $8.40 \pm 1.54$ and $57.97 \pm 4.42$, respectively) were significantly lower than pre-treatment values (mean: $9.21 \pm 2.08$ and $59.42 \pm 4.61$, respectively) ( $p=0.007$ and 0.049 , respectively) (Table 6).
Table 1. Characteristics of patients

\begin{tabular}{|c|c|c|}
\hline Parameter & $N$ & $\%$ \\
\hline \multicolumn{3}{|l|}{ Gender: } \\
\hline Male & 8 & 26.67 \\
\hline Female & 22 & 73.33 \\
\hline \multicolumn{3}{|l|}{ Marital status: } \\
\hline Married & 23 & 76.67 \\
\hline Single/other & 7 & 23.33 \\
\hline \multicolumn{3}{|l|}{ Education status: } \\
\hline Primary school & 6 & 20.00 \\
\hline Middle school & 8 & 26.67 \\
\hline High school & 9 & 30.00 \\
\hline University & 7 & 23.33 \\
\hline \multicolumn{3}{|l|}{ Income status: } \\
\hline Low income & 15 & 50.00 \\
\hline Middle income & 11 & 36.67 \\
\hline High income & 4 & 13.33 \\
\hline Age $^{*}$ & $37.50 \pm 14.14$ & 35.50 \\
\hline Disease duration [months] ${ }^{*}$ & $12.67 \pm 8.05$ & 10.50 \\
\hline Cumulative dose (for each patient) & 1200 & \\
\hline
\end{tabular}

*Results are expressed as mean \pm SD and median data instead of $N$ and $\%$.

Moreover, no significant difference was found between the pre- and post-treatment latency and amplitude values of the motor component of the ulnar nerves $(p>0.05)$. However, post-treatment velocity values (mean: $57.62 \pm 4.13$ ) were significantly lower than pretreatment values (mean: $59.04 \pm 4.02)(p=0.002)$.

In the motor component of the superficial peroneal nerves, post-treatment latency values (mean: $3.90 \pm 0.45$ ) were significantly higher than pre-treatment values

Table 2. Comparison of pre- and post-treatment latency, amplitude, and velocity values of the sensory component of the median nerves

\begin{tabular}{|c|c|c|c|c|c|}
\hline Variable & Mean \pm SD & Median & Minimum & Maximum & $P$-value \\
\hline Latency-pre & $2.17 \pm 0.23$ & 2.13 & 1.75 & 2.75 & $0.278^{b}$ \\
\hline Latency-post & $2.23 \pm 0.31$ & 2.13 & 1.80 & 3.00 & \\
\hline Amplitude-pre & $24.61 \pm 5.55$ & 25.50 & 13.50 & 35.00 & $0.024^{a}$ \\
\hline Amplitude-post & $23.03 \pm 4.71$ & 23.10 & 13.50 & 31.90 & \\
\hline Velocity-pre & $55.67 \pm 5.81$ & 55.60 & 40.00 & 63.40 & $0.821^{b}$ \\
\hline Velocity-post & $55.28 \pm 5.24$ & 55.95 & 42.00 & 63.90 & \\
\hline
\end{tabular}

apaired samples t-test, ${ }^{b}$ Wilcoxon signed-rank test, pre-pre-treatment, post-post-treatment. 
Table 3. Comparison of pre- and post-treatment latency, amplitude, and velocity values of the sensory component of the ulnar nerves

\begin{tabular}{|c|c|c|c|c|c|}
\hline Variable & Mean \pm SD & Median & Minimum & Maximum & $P$-value \\
\hline Latency-pre & $1.89 \pm 0.19$ & 1.90 & 1.50 & 2.45 & $0.436^{\mathrm{b}}$ \\
\hline Latency-post & $1.91 \pm 0.22$ & 1.83 & 1.60 & 2.40 & \\
\hline Amplitude-pre & $20.11 \pm 5.61$ & 18.95 & 11.90 & 34.00 & $0.030^{b}$ \\
\hline Amplitude-post & $18.71 \pm 5.34$ & 17.45 & 12.00 & 31.00 & \\
\hline Velocity-pre & $54.76 \pm 2.99$ & 54.90 & 50.00 & 61.10 & $0.209^{a}$ \\
\hline Velocity-post & $53.94 \pm 3.60$ & 52.75 & 44.90 & 60.60 & \\
\hline
\end{tabular}

${ }^{a}$ Paired samples t-test, ${ }^{b}$ Wilcoxon signed-rank test, pre - pre-treatment, post - post-treatment.

Table 4. Comparison of pre- and post-treatment latency, amplitude, and velocity values of the sensory component of the sural nerves

\begin{tabular}{|c|c|c|c|c|c|}
\hline Variable & Mean \pm SD & Median & Minimum & Maximum & $P$-value \\
\hline Latency-pre & $1.96 \pm 0.21$ & 2.00 & 1.55 & 2.42 & $0.009^{b}$ \\
\hline Latency-post & $2.12 \pm 0.24$ & 2.10 & 1.55 & 2.55 & \\
\hline Amplitude-pre & $15.39 \pm 3.30$ & 14.55 & 9.40 & 22.20 & $0.053^{b}$ \\
\hline Amplitude-post & $14.48 \pm 3.32$ & 14.20 & 8.00 & 21.80 & \\
\hline Velocity-pre & $48.14 \pm 7.09$ & 48.85 & 16.80 & 58.10 & $0.001^{a}$ \\
\hline Velocity-post & $46.34 \pm 3.60$ & 45.80 & 41.90 & 56.90 & \\
\hline
\end{tabular}

${ }^{a}$ Paired samples t-test, ${ }^{b}$ Wilcoxon signed-rank test, pre - pre-treatment, post - post-treatment.

Table 5. Comparison of pre- and post-treatment latency, amplitude, and velocity values of the sensory component of the superficial peroneal nerves

\begin{tabular}{|c|c|c|c|c|c|}
\hline Variable & Mean \pm SD & Median & Minimum & Maximum & $P$-value \\
\hline Latency-pre & $2.00 \pm 0.28$ & 2.00 & 1.50 & 2.55 & 0.497 \\
\hline Latency-post & $2.05 \pm 0.28$ & 2.05 & 1.60 & 2.90 & \\
\hline Amplitude-pre & $16.45 \pm 4.05$ & 16.20 & 9.20 & 23.50 & $<0.001$ \\
\hline Amplitude-post & $14.00 \pm 3.02$ & 14.30 & 9.00 & 21.80 & \\
\hline Velocity-pre & $49.34 \pm 4.61$ & 48.25 & 41.90 & 58.10 & 0.002 \\
\hline Velocity-post & $47.20 \pm 3.36$ & 47.45 & 41.30 & 55.90 & \\
\hline
\end{tabular}

${ }^{a}$ Paired samples t-test, ${ }^{b}$ Wilcoxon signed-rank test, pre - pre-treatment, post - post-treatment.

Table 6. Comparison of pre- and post-treatment latency, amplitude, and velocity values of the motor component of the median nerves

\begin{tabular}{|c|c|c|c|c|c|}
\hline Variable & Mean \pm SD & Median & Minimum & Maximum & $P$-value \\
\hline Latency-pre & $2.76 \pm 0.35$ & 2.73 & 2.15 & 3.70 & $0.052^{b}$ \\
\hline Latency-post & $2.91 \pm 0.47$ & 2.80 & 2.20 & 3.95 & \\
\hline Amplitude-pre & $9.21 \pm 2.08$ & 8.70 & 6.60 & 15.80 & $0.007^{b}$ \\
\hline Amplitude-post & $8.40 \pm 1.54$ & 8.10 & 5.70 & 13.50 & \\
\hline Velocity-pre & $59.42 \pm 4.61$ & 60.15 & 51.00 & 69.40 & $0.049^{a}$ \\
\hline Velocity-post & $57.97 \pm 4.42$ & 58.60 & 50.00 & 64.90 & \\
\hline
\end{tabular}

${ }^{a}$ Paired samples t-test, ${ }^{b}$ Wilcoxon signed-rank test, pre - pre-treatment, post- post-treatment. 
(mean: $3.68 \pm 0.44)(p=0.005)$, whereas post-treatment amplitude and velocity values (mean: $3.47 \pm 0.93$ and $48.35 \pm 4.14$, respectively) were significantly lower than pre-treatment values (mean: $3.98 \pm 1.06$ and $49.64 \pm 3.46$, respectively) ( $p<0.004$ and 0.036 , respectively).

In the motor component of the tibial nerves, no significant difference was found between pre- and posttreatment latency values $(p>0.05)$. However, post-treatment amplitude and velocity values (mean: $7.63 \pm 1.30$ and $44.87 \pm 3.20$, respectively) were significantly lower than pre-treatment values (mean: $8.64 \pm 2.00$ and 46.60 \pm 3.45 , respectively) ( $p<0.002$ and 0.006 , respectively).

\section{Discussion}

The results indicated that omalizumab did not cause peripheral neuropathy but altered the latency, amplitude, and velocity values of the peripheral nerves. To our knowledge, this is the first study in the literature investigating the relationship between omalizumab and peripheral neuropathy.

We evaluated the effect of omalizumab in patients with chronic urticaria. Kim et al. also evaluated the effect of omalizumab in patients with chronic spontaneous urticaria and reported that $61.75 \%$ of the patients were women. Similarly, women also constituted the majority of our patients (73.3\%), which implies that chronic urticaria has a female preponderance [15].

Omalizumab has been shown to be a safe drug in numerous studies. However, a number of side effects have been associated with omalizumab, including anaphylaxis, urticaria, eosinophilic granulomatosis with polyangiitis, susceptibility to parasitic infections, injection site reactions, cardiovascular diseases, and serum sickness [13, 16-20]. On the other hand, a previous study reported that headache and disturbance of sleep were the most common neurological side effects of omalizumab [19]. Similarly, Corren et al. reviewed more than 7,500 patients undergoing omalizumab therapy and found that headache was the most common neurological side effect and also noted that omalizumab led to musculoskeletal disturbances including low back pain, arthralgia, pain in the extremities, and myalgia [17]. In our study, no complaint of headache was found in any patient, which could be ascribed to the small patient series in our study.

Literature reviews indicate that there are a limited number of studies investigating the effect of omalizumab on the nerves. Jachiet et al. reported that omalizumab therapy resulted in optic neuritis in 2 patients [12]. In contrast, Kalteren et al. evaluated a patient with ocular myasthenic syndrome and reported that all the symptoms were resolved after the treatment of the syndrome with omalizumab therapy [21]. On the other hand, Alvarez-Lario et al. evaluated the effectiveness of biological treatment and reported that the side effects of the treatment resulted in peripheral neuropathy associated with Guillain-Barré syndrome in 21 patients. The authors considered that peripheral neuropathy resulted from the increased susceptibility to infections caused by biological agents [22]. As shown in these studies, omalizumab typically increases susceptibility to infections. However, no infection associated with omalizumab was observed in our patients. On the other hand, although no peripheral neuropathy occurred in any of our patients, we consider that omalizumab has the potential to affect peripheral nerves since it has been shown to increase susceptibility to infections, to alter the growth factors and neuropeptides expressed in mast cells, and to trigger the autoimmune process, as observed in a patient described by Kalteren et al. In addition, depending on the finding that omalizumab changed the latency, amplitude, and velocity values of peripheral nerves in our patients, we believe that omalizumab is likely to increase the severity of neuropathy when used in combination with a drug that affects peripheral nerves.

Our study was limited since it was a single-center study and had a relatively small number of patients. Moreover, since we assessed the neurological symptoms of the patients before and three months after the treatment, different outcomes could have been detected if the symptoms had also been assessed at 1 year after the treatment.

\section{Conclusions}

Omalizumab therapy is becoming gradually popular, with a growing side-effect profile. In this study, we investigated the relationship between omalizumab and neuropathy. However, further studies are needed to shed light on our findings.

\section{Conflict of interest}

The authors declare no conflict of interest.

\section{References}

1. Watson JC, Dyck PJ. Peripheral neuropathy: a practical approach to diagnosis and symptom management. Mayo Clin Proc 2015; 90: 940-51.

2. Azhary H, Farooq MU, Bhanushali M, et al. Peripheral neuropathy: differential diagnosis and management. Am Fam Physician 2010; 81: 887-92.

3. Kamel AY, Concepcion O, Schlachterman A, et al. Chronic inflammatory demyelinating polyneuropathy following anti-TNF-alpha therapy with infliximab for Crohn's disease. ACG Case Rep I 2016; 3: 187-9.

4. Nakao M, Asano Y, Nakamura K, et al. The development of chronic inflammatory demyelinating polyneuropathy during adalimumab treatment in a patient with psoriasis vulgaris. Eur J Dermatol 2016; 26: 404-5.

5. Corry DB, Kheradmand F. Induction and regulation of the lgE response. Nature 1999; 402: 18-23. 
6. Lee JH, Lee HY, Jung CG, et al. Therapeutic effect of omalizumab in severe asthma: a real-world study in Korea. Allergy Asthma Immunol Res 2018; 10: 121-30.

7. Maurer M, Metz M, Brehler R, et al. Omalizumab treatment in patients with chronic inducible urticaria: a systematic review of published evidence. J Allergy Clin Immunol 2018; 141: 638-49.

8. Romano C, Sellitto A, De Fanis U, et al. Omalizumab for difficult-to-treat dermatological conditions: clinical and immunological features from a retrospective real-life experience. Clin Drug Investig 2015; 35: 159-68.

9. Johnson D, Krenger W. Interactions of mast cells with the nervous system: recent advances. Neurochem Res 1992; 17: 939-51.

10. van der Kleij HP, Bienenstock J. Significance of conversation between mast cells and nerves. Allergy Asthma Clin Immunol 2005; 1: 65-80.

11. Gouder C, West LM, Montefort S. The real-life clinical effects of 52 weeks of omalizumab therapy for severe persistent allergic asthma. Int J Clin Pharm 2015; 37: 36-43.

12. Jachiet M, Samson M, Cottin V, et al.; French Vasculitis Study Group. Anti-lgE monoclonal antibody (omalizumab) in refractory and relapsing eosinophilic granulomatosis with polyangiitis (Churg-Strauss): data on seventeen patients. Arthritis Rheumatol 2016; 68: 2274-82.

13. Lieberman P, Rahmaoui A, Wong DA. The safety and interpretability of skin tests with omalizumab. Ann Allergy Asthma Immunol 2010; 105: 493-5.

14. Report and recommendations of the San Antonio Conference on Diabetic Neuropathy. American Diabetes Association. Muscle Nerve 1988; 11: 661-7.

15. Kim YS, Park SH, Han K, et al. Clinical course of chronic spontaneous uticaria in the Korean adult population. Allergy Asthma Immunol Res 2018; 10: 83-7.

16. Lin RY, Rodriguez-Baez G, Bhargave GA. Omalizumab-associated anaphylactic reactions reported between January 2007 and June 2008. Ann Allergy Asthma Immunol 2009; 103: 442-5.

17. Corren J, Casale TB, Lanier B, et al. Safety and tolerability of omalizumab. Clin Exp Allergy 2009; 39: 788-97.

18. Cruz AA, Lima F, Sarinho E, et al. Safety of anti-immunoglobulin E therapy with omalizumab in allergic patients at risk of geohelminth infection. Clin Exp Allergy 2007; 37: 197-207.

19. Deniz YM, Gupta N. Safety and tolerability of omalizumab (Xolair), a recombinant humanized monoclonal anti-IgE antibody. Clin Rev Allergy Immunol 2005; 29: 31-48.

20. Iribarren C, Rahmaoui A, Long AA, et al. Cardiovascular and cerebrovascular events among patients receiving omalizumab: results from EXCELS, a prospective cohort study in moderate to severe asthma. J Allergy Clin Immunol 2017; 139: 1489-95.

21. Kalteren WS, Schreurs MWJ, Jorritsma-Smit A, et al. Ocular myasthenic syndrome, adverse reaction to omalizumab? A case report. Br J Clin Pharmacol 2017; 83: 2330-2.

22. Alvarez-Lario B, Prieto-Tejedo R, Colazo-Burlato M, et al. Severe Guillain-Barré syndrome in a patient receiving anti-TNF therapy. Consequence or coincidence. A case-based review. Clin Rheumatol 2013; 32: 1407-12. 